\title{
Satellite Data for Atmospheric Monitoring at the Pierre Auger Observatory
}

\section{Andrew Puyleart ${ }^{a, *}$ on behalf of the Pierre Auger ${ }^{b}$ Collaboration}

(a complete list of authors can be found at the end of the proceedings)

${ }^{a}$ Michigan Technological University, Applied Physics, 1800 Townsend Dr., Houghton MI, USA

${ }^{b}$ Observatorio Pierre Auger, Av. San Martín Norte 304, 5613 Malargüe, Argentina

E-mail: spokespersons@auger.org

Atmospheric monitoring over the $3000 \mathrm{~km}^{2}$ of the Pierre Auger Observatory can be supplemented by satellite data. Methods for night-time cloud detection and aerosol cross-checking were created using the GOES-16 and Aeolus satellites, respectively. The geostationary GOES-16 satellite provides a $100 \%$ up-time view of the cloud cover over the observatory. GOES-13 was used until the end of 2017 for cloud monitoring, but with its retirement a method based on GOES-16 data was developed. The GOES-16 cloud detection method matches the observatory's vertical laser cloud detection method at a rate of $\sim 90 \%$. The Aeolus satellite crosses the Pierre Auger Observatory several times throughout the year firing UV-laser shots. The laser beams leave a track of scattered light in the atmosphere that can be observed by the light sensors of the observatory fluorescence telescopes. Using a parametric model of the aerosol concentration, the laser shots can be reconstructed with different combinations of the aerosol parameters. A minimization procedure then yields the parameter set that best describes the aerosol attenuation. Furthermore, the possibility of studying horizontal homogeneity of aerosols across the array is being investigated.

$3^{\text {th }}$ International Cosmic Ray Conference (ICRC 2021)

July 12th - 23rd, 2021

Online - Berlin, Germany

\footnotetext{
${ }^{*}$ Presenter
} 


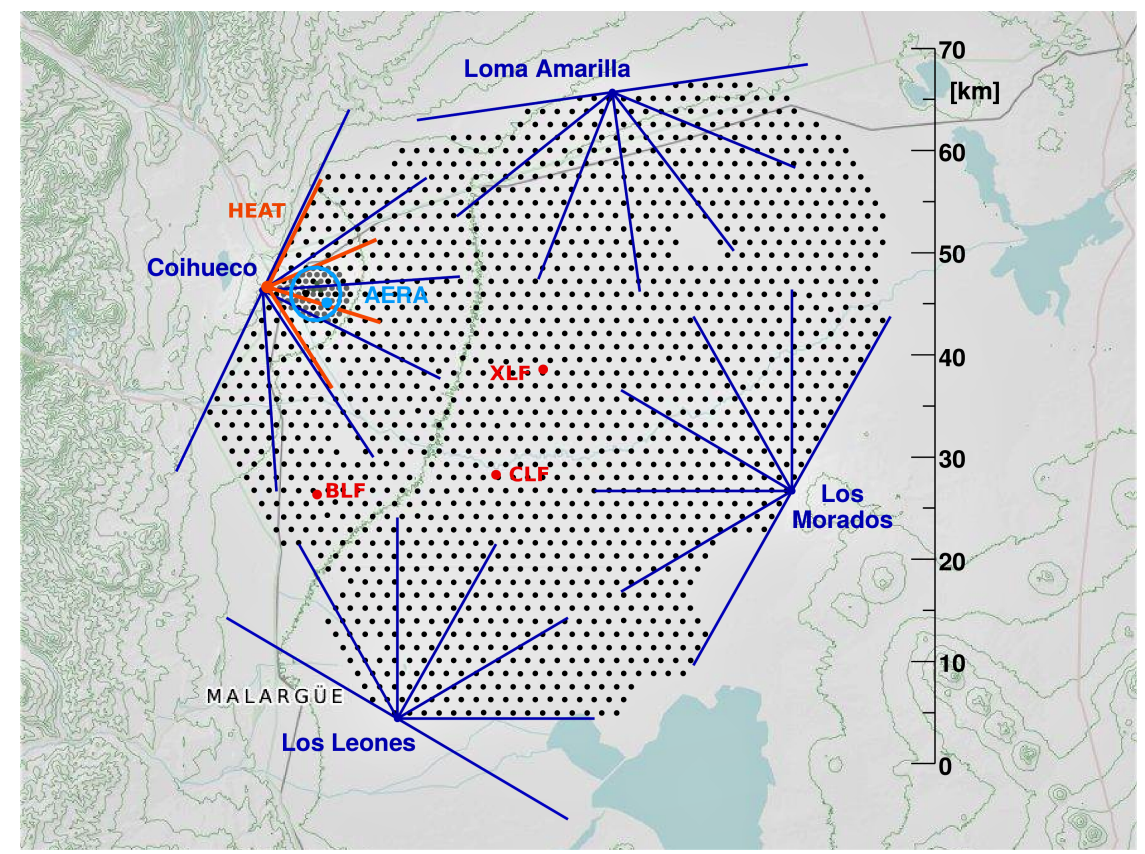

Figure 1: A map of the Pierre Auger Observatory. Water-Cherenkov stations of the surface detector are seen as black dots and the field of view of the fluorescence detector stations are indicated by the blue lines. The high-elevation extension of the fluorescence detector, HEAT, is also seen in orange. The Xtreme and Central Laser Facilities are labelled in red. The balloon launch site and engineering array are also labelled.

\section{Introduction}

The Pierre Auger Observatory is the largest cosmic-ray observatory in the world, covering an area of $3000 \mathrm{~km}^{2}$, see Fig. 1 [1]. The scale of the observatory requires a robust atmospheric monitoring program. In the past, satellites have proven to be a useful tool for atmospheric monitoring [2], [3]. The success of previous satellite tools has led the Pierre Auger collaboration to explore other satellite data as it becomes publicly available. Previously GOES-13 was used to detect cloud cover over the observatory. Cloud cover affects the reconstruction of the energy of the primary cosmic ray particle; if a cloud is present in the path of an extensive air shower the shower cannot be used in analysis. The retirement of GOES-13 made necessary an update to cloud monitoring over the Pierre Auger Observatory; its successor, GOES-16, is now in use as part of atmospheric quality checks during reconstruction of extensive air showers. We will discuss the development of the GOES-16 cloud monitoring algorithm.

The Aeolus satellite crossed the Pierre Auger Observatory several times in 2019. The lasers that were fired by Aeolus may give a unique opportunity to cross-check aerosol concentration measurements for the 2019 operational year. Aerosol concentration is directly related to accurate energy reconstruction of extensive air showers [4] making this cross-check invaluable to confidence in our current processes. 

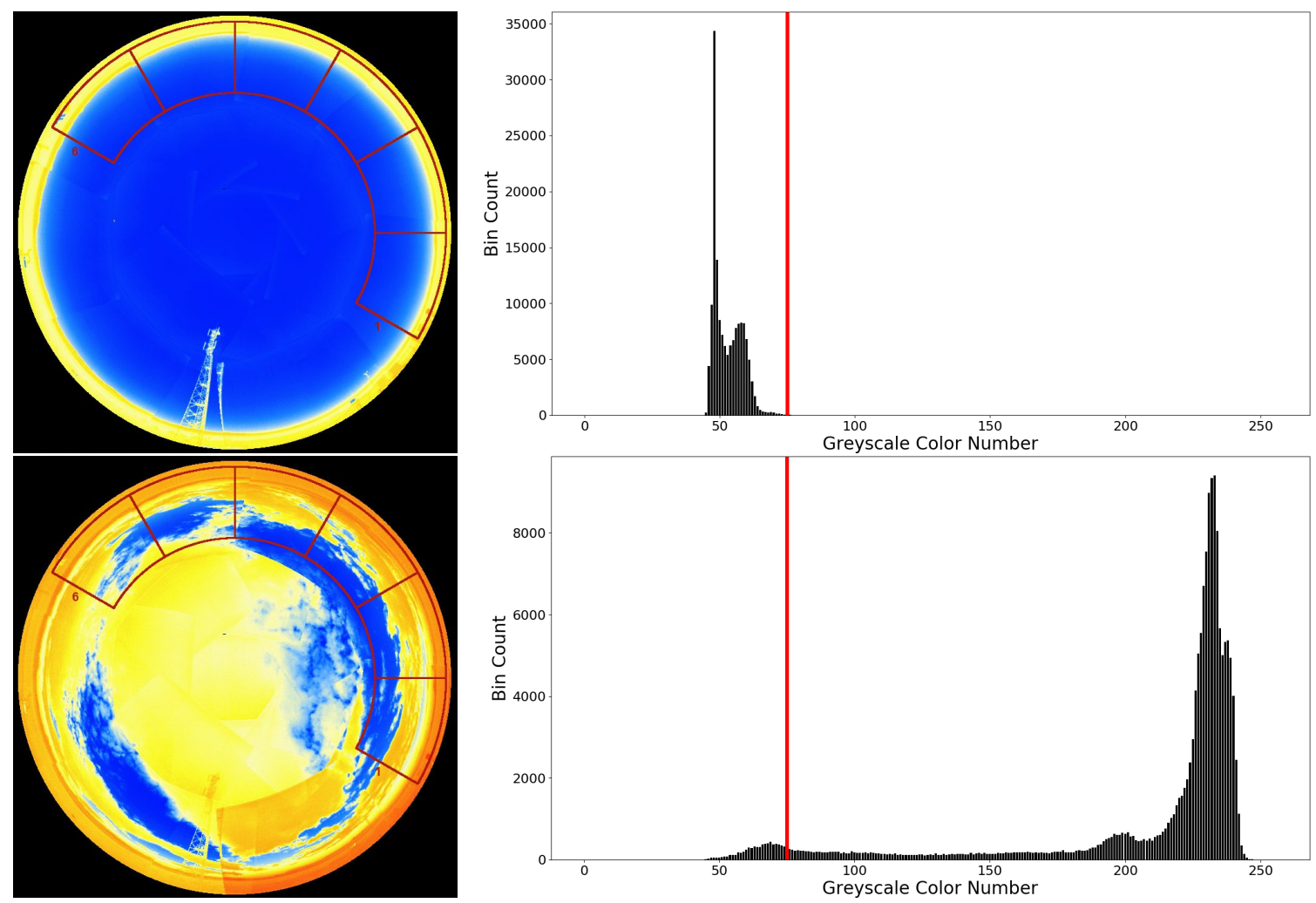

Figure 2: Top: The Los Leones ground IR camera response to a clear sky; its color histogram to the right shows a large response below the color number 75 which is highlighted in red. Bottom: The camera response to a cloudy sky; Here most of the response is seen beyond a color number of 75 .

\section{Cloud Monitoring Using GOES-16}

The fluorescence detector (FD) operates on clear nights with low moonlight illumination; requiring a robust cloud monitoring system [5]. The GOES-16 satellite is a geostationary weather satellite that observes North, as well as South America where the Auger Observatory is located. GOES-16 is equipped with an Advanced Baseline Imager (ABI) camera which has 16 wavebands covering infrared and near-IR wavelengths [6]. To investigate the ABI responses to clear, and cloudy pixels we used a ground IR-camera located at the Los Leones FD site. The ground IR-camera tagged the satellite pixel directly above the camera according to a histogram in a color gradient. Images exhibiting a large cumulative response in color gradients beyond 75 are considered cloudy, see Fig. 2.

Plotting the tagged pixel's brightness temperature response in bands 7, 9, and 14 from the satellite shows a relationship between brightness temperatures and cloudiness, Fig. 3. Using these tagged pixel we applied kernel density estimators (KDE) to the clear and cloudy populations. Combining the value of the two KDEs, and the ratio of clear to cloudy pixels, we use a form of Bayesian probability in equation (1) to give our final cloud probability.

The likelihoods $P(x \mid$ Clear $)$ and $P(x \mid$ Cloud $)$ are the value from the two normalized kernel density functions. The priors, $P$ (Cloud) and $P($ Clear), are the fraction of cloud-tagged and cleartagged points in the 1104-point data-set. Plotting points across the observed region, we obtain the 

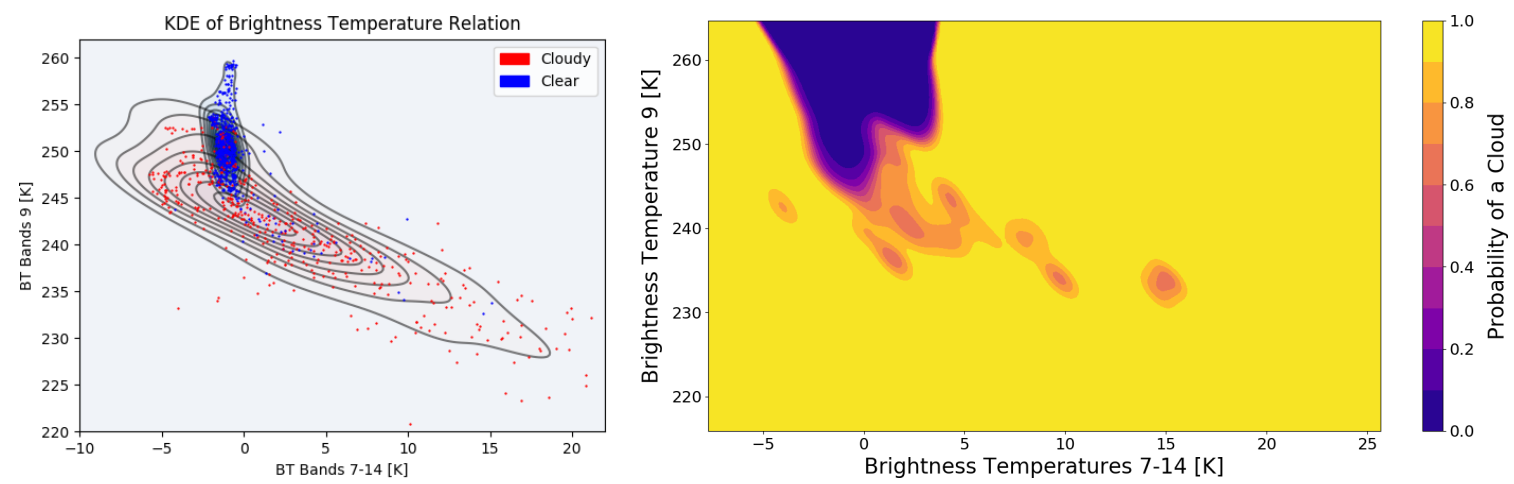

Figure 3: Right: Two KDE contours plotted over pixel scatter plot data. Clear-tagged pixels are in blue, and cloudy in red. Left: The cloud probability map that is produced from the Bayesian probability function.

cloud probability map shown in Fig. 3.

$$
P(\text { Cloud } \mid x)=\frac{P(x \mid \text { Cloud }) P(\text { Cloud })}{P(x \mid \text { Cloud }) P(\text { Cloud })+P(x \mid \text { Clear }) P(\text { Clear })}
$$

To test the goodness of the Bayesian technique we compared it to the National Oceanographic and Atmospheric Administration's (NOAA) Clear-Sky Mask (CSM) product. The CSM is an algorithm using GOES-16 that produces a binary response for cloud coverage of each pixel in an image allowing for a direct comparison to the Bayesian technique [7]. We chose not to use the CSM as our algorithm because the $87 \%$ pixel accuracy of the CSM is not guaranteed beyond $80^{\circ}$ solar zenith angle [8]. The FD of the Pierre Auger Observatory operates only when the solar zenith angle is beyond $70^{\circ}$. Vertical laser shots from the Xtreme Laser Facility (XLF) and the Central Laser Facility (CLF) are routinely recorded by the FD. If a cloud is directly over the XLF or CLF the FD can detect scattered laser light giving their location [9]. We were able to identify the GOES-16 pixels that correspond to the locations of the CLF and XLF. Each image taken by the GOES-16 satellite is matched to the timestamp of vertical laser shots within an eight minute window and its pixel response is extracted. The response of the two satellite techniques and the laser facilities are then compared. Table 1 shows the Bayesian algorithm out performed the CSM by $\sim 10 \%$, and agreeing with the XLF and CLF at a rate of $\sim 90 \%$.

Table 1: Ground truth of Bayesian and Clear-Sky Mask techniques with the XLF and CLF.

\begin{tabular}{r|c|c|c|c} 
& \multicolumn{2}{|c|}{ XLF } & \multicolumn{2}{c}{ CLF } \\
Agree & Bayesian & Clear-Sky Mask & Bayesian & Clear-Sky Mask \\
\cline { 2 - 5 } Disagree & 677 & 258 & 387 & 156 \\
\cline { 2 - 5 } Total & 78 & 68 & 46 & 38 \\
\cline { 2 - 5 } & 755 & 326 & 433 & 194 \\
\cline { 2 - 5 } Percent Agreement & 89.7 & 79.1 & 89.4 & 80.4 \\
\cline { 2 - 5 } False Positives & 39 & 60 & 19 & 28
\end{tabular}



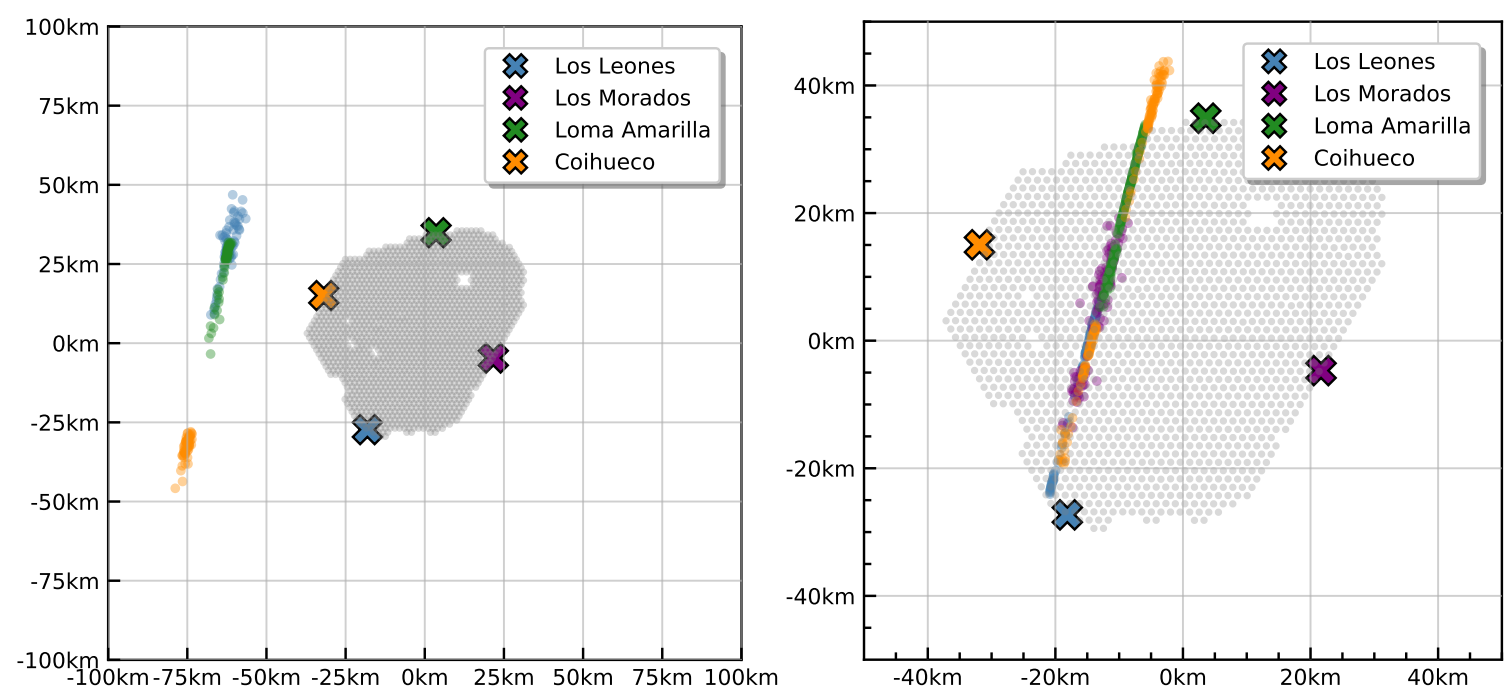

Figure 4: Left: The ground positions of measured laser shots for a transition of the Aeolus main beam compared to the detector array. Fluorescence and surface detector positions are included as a reference. Right: The ground positions of a secondary beam transition.

\section{Parametric Aerosol Concentration Using Aeolus}

Aeolus is the name of a satellite operated by the European Space Agency. Its purpose is the measurement of wind profiles in the atmosphere. Therefore it is equipped with the Atmospheric Laser Doppler Instrument (ALADIN), a UV lidar system, that shoots laser pulses with a wavelength of $354.89 \mathrm{~nm}$ towards Earth at a rate of $50.5 \mathrm{~Hz}$ [10]. Side scattered light from Aeolus laser beams in close proximity to the Pierre Auger Observatory can detected by the fluorescence telescopes, making a reconstruction of the Aeolus laser beams possible. One can differentiate between two kinds of Aeolus lasers. Besides the main beam that is used by Aeolus for its measurements, a secondary beam is observed. It is created by a deliberate reflection of a part of the main beam at the Aeolus transmitter; this avoids a direct illumination of the detection instruments on board of the satellite. Fig. 4 shows two samples of the ground track that is produced by the main beam and the secondary beam respectively. Plotted are the reconstructed ground positions of each measured laser shot for one laser transition from North to South. A laser transition also happens close to the Telescope Array in the Northern hemisphere, which could be beneficial for a direct comparison of the two experiments. However, since these transitions take place during astronomical twilight special precautions would be necessary to allow for an Aeolus measurement at the Telescope Array.

Aeolus is in a sun-synchronous orbit with a repeat cycle of seven days. As a consequence, the laser transition occurs always at the same weekday and time of day, the main beam on Saturdays at Auger at 10:10 UTC and the secondary beam on Fridays at 09:57 UTC. The visibility is however limited by the duty cycle of the fluorescence telescopes. The transition times are close to sunrise, only during winter in the Southern hemisphere are they still within the night time, otherwise, the transition takes place at dawn when no fluorescence telescope measurements are possible. Fig. 5 shows the time of twilight for the Pierre Auger Observatory throughout the year, including the transition times of the main and secondary beam. As can be seen, the visibility is limited to June 


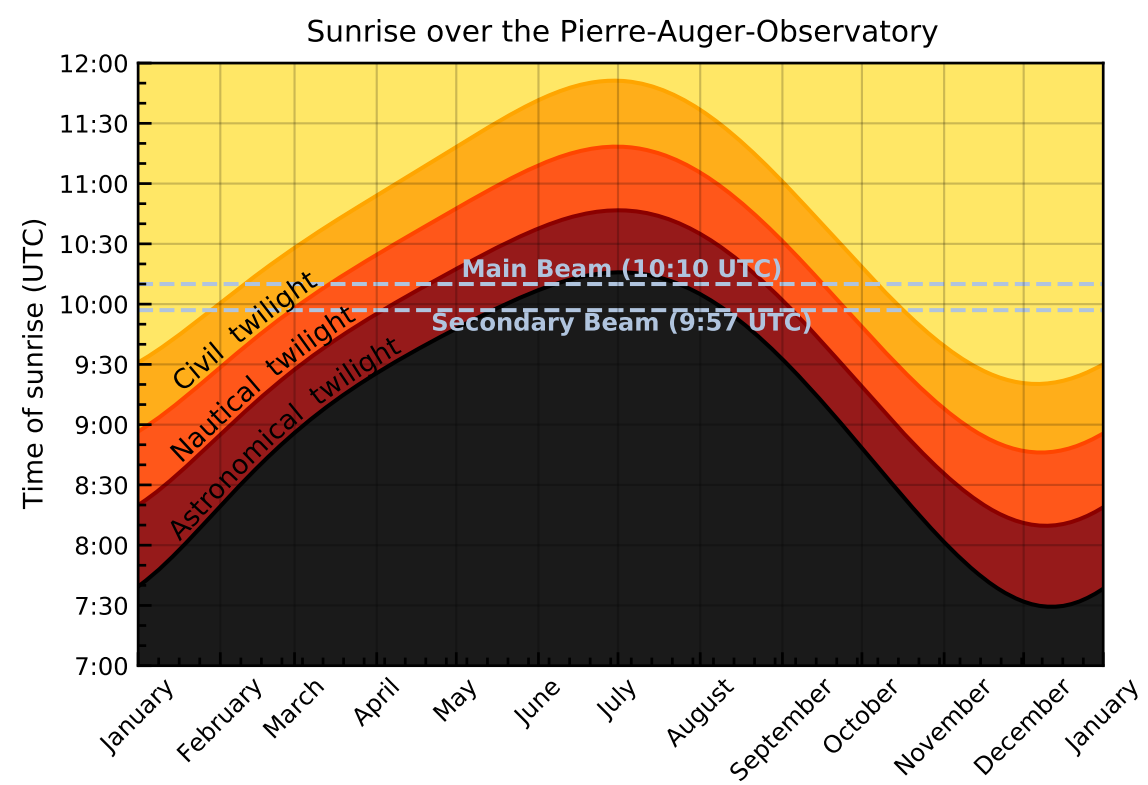

Figure 5: The time of sunrise and different stages of twilight at the Pierre Auger Observatory throughout the year. The horizontal lines mark the transition times of the Aeolus main and secondary beam.

and July for the main beam and mid-May to mid-August for the secondary beam. A detection of the beams is thus only possible for individual nights. In 2019 a total of three main beam and six secondary beam transitions could be detected.

For the extraction of aerosol information from Aeolus laser shots, a parametric model of the aerosol profile is employed. This model assumes an aerosol density that is exponentially decreasing with height. The vertical aerosol optical depth (VAOD) characterizes the amount of light that is lost due to aerosol scattering on a vertical path up to a reference height. It can be described using only the scale height of the exponential decrease and the horizontal aerosol attenuation length at ground level as model parameters. By setting the scale height to a fixed value based on the yearly average, the VAOD and the laser energy are used as free parameters in a likelihood fit. For each measured laser shot and for many points along the beam, the number of photons at the aperture can be compared to the expectation based on an assumed set of parameters. Therefore a likelihood value on the basis of a Poisson statistic can be calculated for the individual combinations of energy and VAOD. The range of different laser-telescope-distances provides leverage on the Aerosol attenuation. The best likelihood value then yields the energy as well as the VAOD. Fig. 6 shows the result of this likelihood scan for one night, corresponding to a main beam transition.

The plot shows the distance to the best fit in units of $\sigma$, as a function of both the laser energy and the VAOD $\tau_{\mathrm{a}}$ at a reference height of $5 \mathrm{~km}$ above ground level. A good statistical precision is reached of $2 \%$ for $\tau_{\mathrm{a}}$ and $0.4 \%$ for the energy. Also drawn in the plot are the expectation of the energy, based on the internal measurement of Aeolus, and a preliminary value for $\tau_{\mathrm{a}}$ measured with the CLF. In this sample night, the Aeolus-based reconstruction is in reasonable agreement with these expectations, within the systematic uncertainty of the energy of $\pm 12 \%$ [11] and of the VAOD of $+32 \%$.

Since Aeolus is extending its mission up until the end of 2022, the study of Aeolus events 


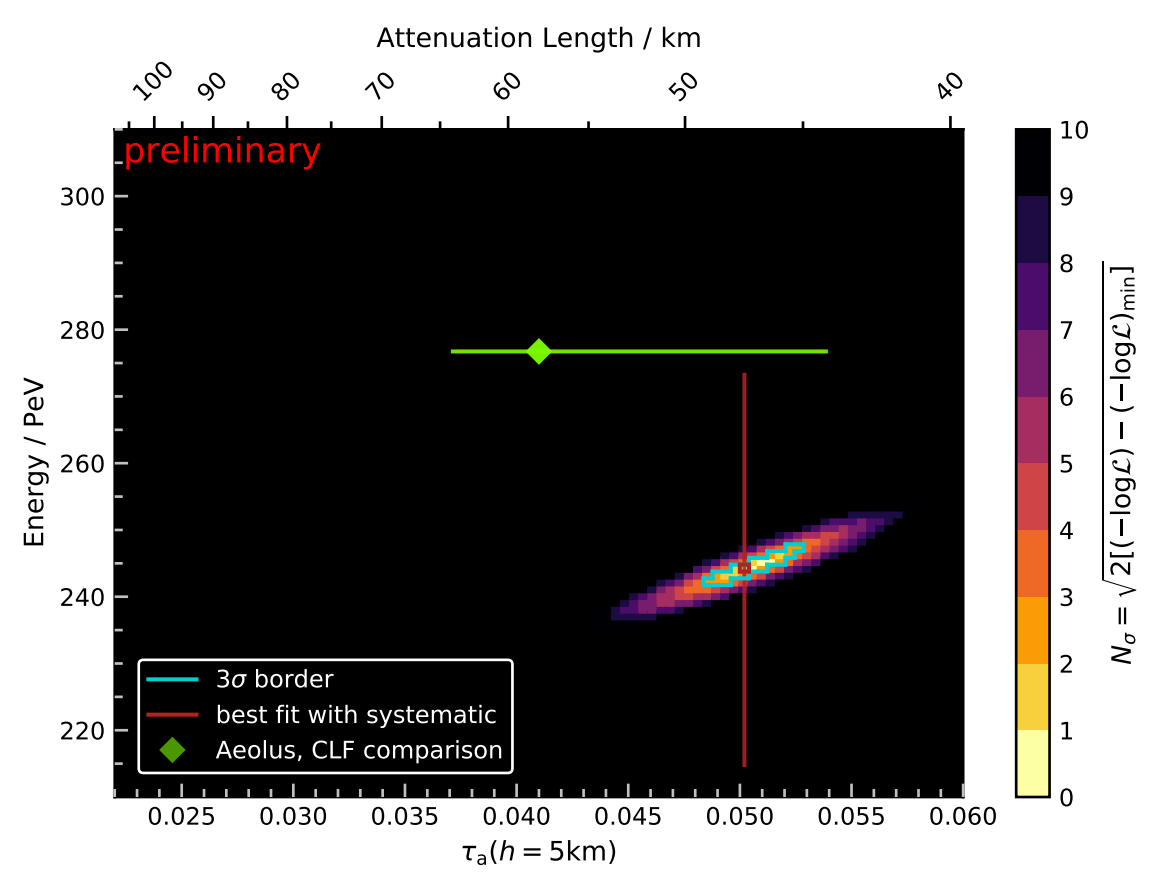

Figure 6: Result of the parameter scan for one main beam transition in August 2019. Colors denote the statistical deviation from the best fit in units of $\sigma$. The nominal Aeolus energy and CLF-determined VAOD are shown by a green diamond. The systematic uncertainty of the VAOD and energy scale are shown as horizontal and vertical lines, respectively.

is continuing with further laser transitions to be measured in the future. An additional promising prospect is a study of horizontal uniformity of the atmospheric conditions. So far this was implicitly assumed in the aerosol model. For a test of this assumption, an approach is to divide the laser ground track into multiple sections and compare the resulting different aerosol attenuation.

With the success of these two and past experiments the Pierre Auger collaboration will continue to explore satellites as supplementary tools in atmospheric monitoring tasks.

Acknowledgements We would like to thank Isabell Krisch and Oliver Reitebuch of DLR for their advice concerning the specifications and operations of the Aeolus satellite.

\section{References}

[1] A. Aab et al. [Pierre Auger Coll.], Nucl. Instrum. Meth. A 798 (2015) 172-213, [1502 . 01323].

[2] P. Abreu et al. [Pierre Auger Coll.], Astropart. Phys. 50-52 (2013) 92-101, [1310 . 1641].

[3] N. Borodai et al. [Pierre Auger Coll.], EPJ. Web Conf. 197 (2019) 02011.

[4] J. Abraham et al. [Pierre Auger Coll.], Astropart. Phys. 33-2 (2010) 108-129, [1002 . 0366].

[5] J. Abraham et al. [Pierre Auger Coll.], Nucl. Instrum. Meth. A 620 (2010) 227-251.

[6] J. Valenti [NOAA], GOES-R Series Product and Users' Guide. 3 (2017) Rev 2.2.

[7] A. Heidinger and W. Straka [NOAA], Algorithm and Theoretical Basis Document. 3 (2013) ABI.

[8] M. Seybold [NOAA], ABI L2+ Clear Sky Mask Beta, Provisional and Full Validation Readiness, Implementation and Management Plan (RIMP), 1 (2016) 0337.

[9] J. Chirinos et al. [Pierre Auger Coll.], EPJ Web Conf. 89 (2015) 03012.

[10] O. Lux et al., Opt. Lett. 45-6 (2020) 1443-1446.

[11] V. Verzi et al. [Pierre Auger Coll.], Proc. 33th ICRC Rio de Janeiro, Brazil (2013), [1307. 5059] 


\section{The Pierre Auger Collaboration}

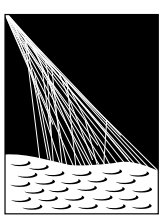

PIERRE

AUSGERVATORY

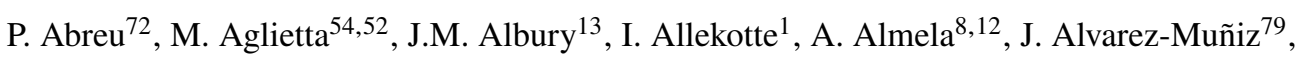
R. Alves Batista ${ }^{80}$, G.A. Anastasi ${ }^{63,52}$, L. Anchordoqui ${ }^{87}$, B. Andrada ${ }^{8}$, S. Andringa ${ }^{72}$, C. $\mathrm{Aramo}^{50}$, P.R. Araújo Ferreira ${ }^{42}$, J. C. Arteaga Velázquez ${ }^{67}$, H. Asorey $^{8}$, P. Assis ${ }^{72}$, G. Avila ${ }^{11}$, A.M. Badescu ${ }^{75}$, A. Bakalova ${ }^{32}$, A. Balaceanu ${ }^{73}$, F. Barbato ${ }^{45,46}$, R.J. Barreira Luz $^{72}$, K.H. Becker ${ }^{38}$, J.A. Bellido ${ }^{13,69}$, C. Berat ${ }^{36}$, M.E. Bertaina ${ }^{63,52}$, X. Bertou ${ }^{1}$, P.L. Biermann ${ }^{b}$, V. Binet ${ }^{6}$, K. Bismark ${ }^{39,8}$, T. Bister ${ }^{42}$, J. Biteau ${ }^{37}$, J. Blazek ${ }^{32}$, C. Bleve ${ }^{36}$, M. Boháčová ${ }^{32}$, D. Boncioli ${ }^{57,46}$, C. Bonifazi ${ }^{9,26}$, L. Bonneau Arbeletche ${ }^{21}$, N. Borodai ${ }^{70}$, A.M. Botti ${ }^{8}$, J. Brack ${ }^{d}$, T. Bretz ${ }^{42}$, P.G. Brichetto Orchera ${ }^{8}$, F.L. Briechle ${ }^{42}$, P. Buchholz ${ }^{44}$, A. Bueno ${ }^{78}$, S. Buitink ${ }^{15}$, M. Buscemi ${ }^{47}$, M. Büsken ${ }^{39,8}$, K.S. Caballero-Mora ${ }^{66}$, L. Caccianiga ${ }^{59,49}$, F. Canfora ${ }^{80,81}$, I. Caracas ${ }^{38}$, J.M. Carceller ${ }^{78}$, R. Caruso ${ }^{58,47}$, A. Castellina ${ }^{54,52}$, F. Catalani ${ }^{19}$, G. Cataldi ${ }^{48}$, L. Cazon ${ }^{72}$, M. Cerda ${ }^{10}$, J.A. Chinellato ${ }^{22}$, J. Chudoba ${ }^{32}$, L. Chytka ${ }^{33}$, R.W. Clay ${ }^{13}$, A.C. Cobos Ceruttii ${ }^{7}$, R. Colalillo ${ }^{60,50}$, A. Coleman ${ }^{93}$, M.R. Coluccia ${ }^{48}$, R. Conceição ${ }^{72}$, A. Condorelli ${ }^{45,46}$, G. Consolati ${ }^{49,55}$, F. Contreras ${ }^{11}$, F. Convenga ${ }^{56,48}$, D. Correia dos Santos $^{28}$, C.E. Covault ${ }^{85}$, S. Dasso ${ }^{5,3}$, K. Daumiller ${ }^{41}$, B.R. Dawson ${ }^{13}$, J.A. Day ${ }^{13}$, R.M. de Almeida $^{28}$, J. de Jesús ${ }^{8,41}$, S.J. de Jong ${ }^{80,81}$, G. De Mauro ${ }^{80,81}$, J.R.T. de Mello Neto ${ }^{26,27}$, I. De Mitri ${ }^{45,46}$, J. de Oliveira ${ }^{18}$, D. de Oliveira Franco ${ }^{22}$, F. de Palma ${ }^{56,48}$, V. de Souza $^{20}$, E. De Vito ${ }^{56,48}$, M. del Río ${ }^{11}$, O. Deligny ${ }^{34}$, L. Deval ${ }^{41,8}$, A. di Matteo $^{52}$, C. Dobrigkeit ${ }^{22}$, J.C. D’Olivo ${ }^{68}$, L.M. Domingues Mendes ${ }^{72}$, R.C. dos Anjos ${ }^{25}$, D. dos Santos $^{28}$, M.T. Dova ${ }^{4}$, J. Ebr ${ }^{32}$, R. Engel ${ }^{39,41}$, I. Epicoco ${ }^{56,48}$, M. Erdmann ${ }^{42}$, C.O. Escobar ${ }^{a}$, A. Etchegoyen ${ }^{8,12}$, H. Falcke ${ }^{80,82,81}$, J. Farmer ${ }^{92}$, G. Farrar ${ }^{90}$, A.C. Fauth ${ }^{22}$, N. Fazzini ${ }^{a}$, F. Feldbusch ${ }^{40}$, F. Fenu ${ }^{54,52}$,

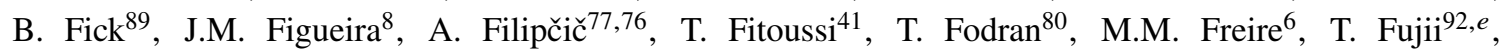
A. Fuster $^{8,12}$, C. Galea ${ }^{80}$, C. Galelli ${ }^{59,49}$, B. García ${ }^{7}$, A.L. Garcia Vegas ${ }^{42}$, H. Gemmeke ${ }^{40}$, F. Gesualdi ${ }^{8,41}$, A. Gherghel-Lascu ${ }^{73}$, P.L. Ghia ${ }^{34}$, U. Giaccari ${ }^{80}$, M. Giammarchi ${ }^{49}$, J. Glombitza ${ }^{42}$, F. Gobbi ${ }^{10}$, F. Gollan ${ }^{8}$, G. Golup ${ }^{1}$, M. Gómez Berisso ${ }^{1}$, P.F. Gómez Vitale ${ }^{11}$, J.P. Gongora ${ }^{11}$, J.M. González ${ }^{1}$, N. González ${ }^{14}$, I. Goos ${ }^{1,41}$, D. Góra ${ }^{70}$, A. Gorgi ${ }^{54,52}$, M. Gottowik ${ }^{38}$, T.D. Grubb ${ }^{13}$, F. Guarino ${ }^{60,50}$, G.P. Guedes ${ }^{23}$, E. Guido ${ }^{52,63}$, S. $\mathrm{Hahn}^{41,8}$, P. $\mathrm{Hamal}^{32}$, M.R. Hampel ${ }^{8}$, P. Hansen ${ }^{4}$, D. Harari ${ }^{1}$, V.M. Harvey ${ }^{13}$, A. Haungs ${ }^{41}$, T. Hebbeker ${ }^{42}$, D. Heck ${ }^{41}$, G.C. Hill ${ }^{13}$, C. Hojvat ${ }^{a}$, J.R. Hörandel ${ }^{80,81}$, P. Horvath ${ }^{33}$, M. Hrabovský ${ }^{33}$, T. Huege ${ }^{41,15}$, A. Insolia ${ }^{58,47}$, P.G. Isar $^{74}$, P. Janecek ${ }^{32}$, J.A. Johnsen ${ }^{86}$, J. Jurysek ${ }^{32}$, A. Kääpä ${ }^{38}$, K.H. Kampert ${ }^{38}$, N. Karastathis ${ }^{41}$, B. Keilhauer ${ }^{41}$, J. Kemp ${ }^{42}$, A. Khakurdikar ${ }^{80}$, V.V. Kizakke Covilakam $^{8,41}$, H.O. Klages ${ }^{41}$, M. Kleifges ${ }^{40}$, J. Kleinfeller ${ }^{10}$, M. Köpke ${ }^{39}$, N. Kunka ${ }^{40}$, B.L. Lago ${ }^{17}$, R.G. Lang ${ }^{20}$, N. Langner ${ }^{42}$, M.A. Leigui de Oliveira ${ }^{24}$, V. Lenok $^{41}$, A. Letessier-Selvon ${ }^{35}$, I. LhenryYvon $^{34}$, D. Lo Presti ${ }^{58,47}$, L. Lopes ${ }^{72}$, R. López ${ }^{64}$, L. Lu ${ }^{94}$, Q. Luce ${ }^{39}$, J.P. Lundquist ${ }^{76}$, A. Machado Payeras $^{22}$, G. Mancarella ${ }^{56,48}$, D. Mandat ${ }^{32}$, B.C. Manning ${ }^{13}$, J. Manshanden ${ }^{43}$, P. Mantsch ${ }^{a}$, S. Marafico ${ }^{34}$, A.G. Mariazzi ${ }^{4}$, I.C. Mariş ${ }^{14}$, G. Marsella ${ }^{61,47}$, D. Martello ${ }^{56,48}$, S. Martinelli ${ }^{41,8}$, O. Martínez Bravo ${ }^{64}$, M. Mastrodicasa ${ }^{57,46}$, H.J. Mathes ${ }^{41}$, J. Matthews ${ }^{88}$, G. Matthiae ${ }^{62,51}$, E. Mayotte ${ }^{38}$, P.O. Mazur ${ }^{a}$, G. MedinaTanco $^{68}$, D. Melo $^{8}$, A. Menshikov ${ }^{40}$, K.-D. Merenda ${ }^{86}$, S. Michal ${ }^{33}$, M.I. Micheletti ${ }^{6}$, L. Miramonti ${ }^{59,49}$, S. Mollerach ${ }^{1}$, F. Montanet ${ }^{36}$, C. Morello ${ }^{54,52}$, M. Mostafá ${ }^{91}$, A.L. Müller ${ }^{8}$, M.A. Muller ${ }^{22}$, K. Mulrey ${ }^{15}$, R. Mussa ${ }^{52}$, M. Muzio ${ }^{90}$, W.M. Namasaka ${ }^{38}$, A. Nasr-Esfahani ${ }^{38}$, L. Nellen ${ }^{68}$, M. Niculescu-Oglinzanu ${ }^{73}$, M. Niechciol ${ }^{44}$, D. Nitz ${ }^{89}$, D. Nosek ${ }^{31}$, V. Novotny ${ }^{31}$, L. Nožka ${ }^{33}$, A Nucita ${ }^{56,48}$, L.A. Núñez ${ }^{30}$, M. Palatka ${ }^{32}$, J. Pallotta ${ }^{2}$, P. Papenbreer ${ }^{38}$, G. Parente ${ }^{79}$, A. Parra ${ }^{64}$, J. Pawlowsky ${ }^{38}$, M. Pech ${ }^{32}$, F. Pedreira ${ }^{79}$, J. Pȩkala ${ }^{70}$, R. Pelayo ${ }^{65}$, J. Peña-Rodriguez ${ }^{30}$, E.E. Pereira Martins ${ }^{39,8}$, J. Perez Armand ${ }^{21}$, C. Pérez Bertolli $^{8,41}$, M. Perlin ${ }^{8,41}$, L. Perrone ${ }^{56,48}$, S. Petrera ${ }^{45,46}$, T. Pierog ${ }^{41}$, M. Pimenta ${ }^{72}$, V. Pirronello ${ }^{58,47}$, M. Platino ${ }^{8}$, B. Pont $^{80}$, M. Pothast ${ }^{81,80}$, P. Privitera ${ }^{92}$, M. Prouza ${ }^{32}$, A. Puyleart ${ }^{89}$, S. Querchfeld ${ }^{38}$, J. Rautenberg ${ }^{38}$, D. Ravignani ${ }^{8}$, M. Reininghaus ${ }^{41,8}$, J. Ridky ${ }^{32}$, F. Riehn ${ }^{72}$, M. Risse ${ }^{44}$, V. Rizi ${ }^{57,46}$, W. Rodrigues de Carvalho ${ }^{21}$, J. Rodriguez Rojo ${ }^{11}$, M.J. Roncoroni ${ }^{8}$, S. Rossoni ${ }^{43}$, M. Roth ${ }^{41}$, E. Roulet ${ }^{1}$, A.C. Rovero ${ }^{5}$, P. Ruehl ${ }^{44}$, A. Saftoiu ${ }^{73}$, F. Salamida ${ }^{57,46}$, H. Salazar ${ }^{64}$, G. Salina ${ }^{51}$, J.D. Sanabria Gomez ${ }^{30}$, F. Sánchez ${ }^{8}$, E.M. Santos ${ }^{21}$, E. Santos ${ }^{32}$, F. Sarazin ${ }^{86}$, R. Sarmento ${ }^{72}$, C. Sarmiento-Cano ${ }^{8}$, R. Sato ${ }^{11}$, 
P. Savina ${ }^{56,48,34,94}$, C.M. Schäfer ${ }^{41}$, V. Scherini ${ }^{56,48}$, H. Schieler ${ }^{41}$, M. Schimassek ${ }^{39,8}$, M. Schimp ${ }^{38}$, F. Schlüter ${ }^{41,8}$, D. Schmidt ${ }^{39}$, O. Scholten ${ }^{84,15}$, P. Schovánek ${ }^{32}$, F.G. Schröder ${ }^{93,41}$, S. Schröder ${ }^{38}$, J. Schulte ${ }^{42}$, S.J. Sciutto ${ }^{4}$, M. Scornavacche ${ }^{8,41}$, A. Segreto ${ }^{53,47}$, S. Sehgal ${ }^{38}$, R.C. Shellard ${ }^{16}$, G. Sigl ${ }^{43}$, G. Silli ${ }^{8,41}$, O. Sima ${ }^{73, f}$, R. Šmída ${ }^{92}$, P. Sommers ${ }^{91}$, J.F. Soriano ${ }^{87}$, J. Souchard ${ }^{36}$, R. Squartini ${ }^{10}$, M. Stadelmaier ${ }^{41,8}$, D. Stanca ${ }^{73}$, S. Stanič ${ }^{76}$, J. Stasielak ${ }^{70}$, P. Stassi ${ }^{36}$, A. Streich ${ }^{39,8}$, M. Suárez-Durán ${ }^{14}$, T. Sudholz ${ }^{13}$, T. Suomijärvi ${ }^{37}$, A.D. Supanitsky ${ }^{8}$, Z. Szadkowski ${ }^{71}$, A. Tapia ${ }^{29}$, C. Taricco ${ }^{63,52}$, C. Timmermans ${ }^{81,80}$, O. Tkachenko ${ }^{41}$, P. Tobiska ${ }^{32}$, C.J. Todero Peixoto ${ }^{19}$, B. Tomé ${ }^{72}$, Z. Torrès ${ }^{36}$, A. Travaini ${ }^{10}$, P. Travnicek $^{32}$, C. Trimarelli ${ }^{57,46}$, M. Tueros ${ }^{4}$, R. Ulrich ${ }^{41}$, M. Unger ${ }^{41}$, L. Vaclavek ${ }^{33}$, M. Vacula ${ }^{33}$, J.F. Valdés Galicia ${ }^{68}$, L. Valore ${ }^{60,50}$, E. Varela ${ }^{64}$, A. Vásquez-Ramírez ${ }^{30}$, D. Veberič ${ }^{41}$, C. Ventura ${ }^{27}$, I.D. Vergara Quispe ${ }^{4}$, V. Verzi ${ }^{51}$, J. Vicha ${ }^{32}$, J. Vink $^{83}$, S. Vorobiov ${ }^{76}$, H. Wahlberg ${ }^{4}$, C. Watanabe ${ }^{26}$, A.A. Watson ${ }^{c}$, M. Weber ${ }^{40}$, A. Weindl ${ }^{41}$, L. Wiencke ${ }^{86}$, H. Wilczyński ${ }^{70}$, M. Wirtz ${ }^{42}$, D. Wittkowski ${ }^{38}$, B. Wundheiler ${ }^{8}$, A. Yushkov $^{32}$, O. Zapparrata ${ }^{14}$, E. Zas ${ }^{79}$, D. Zavrtanik ${ }^{76,77}$, M. Zavrtanik ${ }^{77,76}$, L. Zehrer ${ }^{76}$

${ }^{1}$ Centro Atómico Bariloche and Instituto Balseiro (CNEA-UNCuyo-CONICET), San Carlos de Bariloche, Argentina

${ }^{2}$ Centro de Investigaciones en Láseres y Aplicaciones, CITEDEF and CONICET, Villa Martelli, Argentina

${ }^{3}$ Departamento de Física and Departamento de Ciencias de la Atmósfera y los Océanos, FCEyN, Universidad de Buenos Aires and CONICET, Buenos Aires, Argentina

${ }^{4}$ IFLP, Universidad Nacional de La Plata and CONICET, La Plata, Argentina

${ }^{5}$ Instituto de Astronomía y Física del Espacio (IAFE, CONICET-UBA), Buenos Aires, Argentina

${ }^{6}$ Instituto de Física de Rosario (IFIR) - CONICET/U.N.R. and Facultad de Ciencias Bioquímicas y Farmacéuticas U.N.R., Rosario, Argentina

${ }^{7}$ Instituto de Tecnologías en Detección y Astropartículas (CNEA, CONICET, UNSAM), and Universidad Tecnológica Nacional - Facultad Regional Mendoza (CONICET/CNEA), Mendoza, Argentina

${ }^{8}$ Instituto de Tecnologías en Detección y Astropartículas (CNEA, CONICET, UNSAM), Buenos Aires, Argentina

${ }^{9}$ International Center of Advanced Studies and Instituto de Ciencias Físicas, ECyT-UNSAM and CONICET, Campus Miguelete - San Martín, Buenos Aires, Argentina

${ }^{10}$ Observatorio Pierre Auger, Malargüe, Argentina

${ }^{11}$ Observatorio Pierre Auger and Comisión Nacional de Energía Atómica, Malargüe, Argentina

12 Universidad Tecnológica Nacional - Facultad Regional Buenos Aires, Buenos Aires, Argentina

${ }^{13}$ University of Adelaide, Adelaide, S.A., Australia

14 Université Libre de Bruxelles (ULB), Brussels, Belgium

15 Vrije Universiteit Brussels, Brussels, Belgium

${ }^{16}$ Centro Brasileiro de Pesquisas Fisicas, Rio de Janeiro, RJ, Brazil

${ }^{17}$ Centro Federal de Educação Tecnológica Celso Suckow da Fonseca, Nova Friburgo, Brazil

${ }^{18}$ Instituto Federal de Educação, Ciência e Tecnologia do Rio de Janeiro (IFRJ), Brazil

${ }^{19}$ Universidade de São Paulo, Escola de Engenharia de Lorena, Lorena, SP, Brazil

${ }^{20}$ Universidade de São Paulo, Instituto de Física de São Carlos, São Carlos, SP, Brazil

${ }^{21}$ Universidade de São Paulo, Instituto de Física, São Paulo, SP, Brazil

${ }^{22}$ Universidade Estadual de Campinas, IFGW, Campinas, SP, Brazil

${ }^{23}$ Universidade Estadual de Feira de Santana, Feira de Santana, Brazil

${ }^{24}$ Universidade Federal do ABC, Santo André, SP, Brazil

${ }^{25}$ Universidade Federal do Paraná, Setor Palotina, Palotina, Brazil

${ }^{26}$ Universidade Federal do Rio de Janeiro, Instituto de Física, Rio de Janeiro, RJ, Brazil

${ }^{27}$ Universidade Federal do Rio de Janeiro (UFRJ), Observatório do Valongo, Rio de Janeiro, RJ, Brazil

${ }^{28}$ Universidade Federal Fluminense, EEIMVR, Volta Redonda, RJ, Brazil

${ }^{29}$ Universidad de Medellín, Medellín, Colombia

${ }^{30}$ Universidad Industrial de Santander, Bucaramanga, Colombia

${ }^{31}$ Charles University, Faculty of Mathematics and Physics, Institute of Particle and Nuclear Physics, Prague, Czech Republic

32 Institute of Physics of the Czech Academy of Sciences, Prague, Czech Republic 
33 Palacky University, RCPTM, Olomouc, Czech Republic

34 CNRS/IN2P3, IJCLab, Université Paris-Saclay, Orsay, France

${ }^{35}$ Laboratoire de Physique Nucléaire et de Hautes Energies (LPNHE), Sorbonne Université, Université de Paris, CNRSIN2P3, Paris, France

${ }^{36}$ Univ. Grenoble Alpes, CNRS, Grenoble Institute of Engineering Univ. Grenoble Alpes, LPSC-IN2P3, 38000 Grenoble, France

${ }^{37}$ Université Paris-Saclay, CNRS/IN2P3, IJCLab, Orsay, France

38 Bergische Universität Wuppertal, Department of Physics, Wuppertal, Germany

${ }^{39}$ Karlsruhe Institute of Technology (KIT), Institute for Experimental Particle Physics, Karlsruhe, Germany

${ }^{40}$ Karlsruhe Institute of Technology (KIT), Institut für Prozessdatenverarbeitung und Elektronik, Karlsruhe, Germany

${ }^{41}$ Karlsruhe Institute of Technology (KIT), Institute for Astroparticle Physics, Karlsruhe, Germany

${ }^{42}$ RWTH Aachen University, III. Physikalisches Institut A, Aachen, Germany

43 Universität Hamburg, II. Institut für Theoretische Physik, Hamburg, Germany

${ }^{44}$ Universität Siegen, Department Physik - Experimentelle Teilchenphysik, Siegen, Germany

${ }^{45}$ Gran Sasso Science Institute, L'Aquila, Italy

46 INFN Laboratori Nazionali del Gran Sasso, Assergi (L'Aquila), Italy

${ }^{47}$ INFN, Sezione di Catania, Catania, Italy

${ }^{48}$ INFN, Sezione di Lecce, Lecce, Italy

${ }^{49}$ INFN, Sezione di Milano, Milano, Italy

${ }^{50}$ INFN, Sezione di Napoli, Napoli, Italy

${ }^{51}$ INFN, Sezione di Roma "Tor Vergata", Roma, Italy

52 INFN, Sezione di Torino, Torino, Italy

53 Istituto di Astrofisica Spaziale e Fisica Cosmica di Palermo (INAF), Palermo, Italy

54 Osservatorio Astrofisico di Torino (INAF), Torino, Italy

55 Politecnico di Milano, Dipartimento di Scienze e Tecnologie Aerospaziali , Milano, Italy

56 Università del Salento, Dipartimento di Matematica e Fisica "E. De Giorgi”, Lecce, Italy

57 Università dell'Aquila, Dipartimento di Scienze Fisiche e Chimiche, L'Aquila, Italy

58 Università di Catania, Dipartimento di Fisica e Astronomia, Catania, Italy

${ }^{59}$ Università di Milano, Dipartimento di Fisica, Milano, Italy

${ }^{60}$ Università di Napoli "Federico II", Dipartimento di Fisica "Ettore Pancini”, Napoli, Italy

${ }^{61}$ Università di Palermo, Dipartimento di Fisica e Chimica "E. Segrè", Palermo, Italy

62 Università di Roma "Tor Vergata", Dipartimento di Fisica, Roma, Italy

${ }^{63}$ Università Torino, Dipartimento di Fisica, Torino, Italy

${ }^{64}$ Benemérita Universidad Autónoma de Puebla, Puebla, México

${ }^{65}$ Unidad Profesional Interdisciplinaria en Ingeniería y Tecnologías Avanzadas del Instituto Politécnico Nacional (UPIITA-IPN), México, D.F., México

66 Universidad Autónoma de Chiapas, Tuxtla Gutiérrez, Chiapas, México

${ }^{67}$ Universidad Michoacana de San Nicolás de Hidalgo, Morelia, Michoacán, México

${ }^{68}$ Universidad Nacional Autónoma de México, México, D.F., México

${ }^{69}$ Universidad Nacional de San Agustin de Arequipa, Facultad de Ciencias Naturales y Formales, Arequipa, Peru

${ }^{70}$ Institute of Nuclear Physics PAN, Krakow, Poland

${ }^{71}$ University of Łódź, Faculty of High-Energy Astrophysics, Łódź, Poland

${ }^{72}$ Laboratório de Instrumentação e Física Experimental de Partículas - LIP and Instituto Superior Técnico - IST, Universidade de Lisboa - UL, Lisboa, Portugal

73 "Horia Hulubei” National Institute for Physics and Nuclear Engineering, Bucharest-Magurele, Romania

${ }^{74}$ Institute of Space Science, Bucharest-Magurele, Romania

75 University Politehnica of Bucharest, Bucharest, Romania

76 Center for Astrophysics and Cosmology (CAC), University of Nova Gorica, Nova Gorica, Slovenia

${ }^{77}$ Experimental Particle Physics Department, J. Stefan Institute, Ljubljana, Slovenia

78 Universidad de Granada and C.A.F.P.E., Granada, Spain

${ }^{79}$ Instituto Galego de Física de Altas Enerxías (IGFAE), Universidade de Santiago de Compostela, Santiago de Compostela, Spain 
${ }^{80}$ IMAPP, Radboud University Nijmegen, Nijmegen, The Netherlands

${ }^{81}$ Nationaal Instituut voor Kernfysica en Hoge Energie Fysica (NIKHEF), Science Park, Amsterdam, The Netherlands

82 Stichting Astronomisch Onderzoek in Nederland (ASTRON), Dwingeloo, The Netherlands

${ }^{83}$ Universiteit van Amsterdam, Faculty of Science, Amsterdam, The Netherlands

${ }^{84}$ University of Groningen, Kapteyn Astronomical Institute, Groningen, The Netherlands

85 Case Western Reserve University, Cleveland, OH, USA

86 Colorado School of Mines, Golden, CO, USA

${ }^{87}$ Department of Physics and Astronomy, Lehman College, City University of New York, Bronx, NY, USA

${ }^{88}$ Louisiana State University, Baton Rouge, LA, USA

${ }^{89}$ Michigan Technological University, Houghton, MI, USA

${ }^{90}$ New York University, New York, NY, USA

${ }^{91}$ Pennsylvania State University, University Park, PA, USA

92 University of Chicago, Enrico Fermi Institute, Chicago, IL, USA

93 University of Delaware, Department of Physics and Astronomy, Bartol Research Institute, Newark, DE, USA

94 University of Wisconsin-Madison, Department of Physics and WIPAC, Madison, WI, USA

${ }^{a}$ Fermi National Accelerator Laboratory, Fermilab, Batavia, IL, USA

${ }^{b}$ Max-Planck-Institut für Radioastronomie, Bonn, Germany

${ }^{c}$ School of Physics and Astronomy, University of Leeds, Leeds, United Kingdom

${ }^{d}$ Colorado State University, Fort Collins, CO, USA

$e^{e}$ now at Hakubi Center for Advanced Research and Graduate School of Science, Kyoto University, Kyoto, Japan

$f$ also at University of Bucharest, Physics Department, Bucharest, Romania 